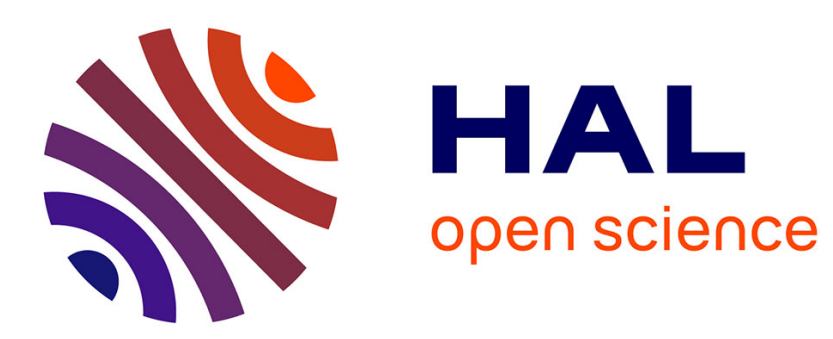

\title{
La dimension politique dans l'approche de la proximité
}

Damien Talbot

\section{To cite this version:}

Damien Talbot. La dimension politique dans l'approche de la proximité. Géographie, Économie, Société, 2010, 12 (2), pp.125-144. 10.3166/ges.12.125-144 . hal-02364226

\section{HAL Id: hal-02364226 \\ https://hal.science/hal-02364226}

Submitted on 14 Nov 2019

HAL is a multi-disciplinary open access archive for the deposit and dissemination of scientific research documents, whether they are published or not. The documents may come from teaching and research institutions in France or abroad, or from public or private research centers.
L'archive ouverte pluridisciplinaire HAL, est destinée au dépôt et à la diffusion de documents scientifiques de niveau recherche, publiés ou non, émanant des établissements d'enseignement et de recherche français ou étrangers, des laboratoires publics ou privés. 


\title{
LA DIMENSION POLITIQUE DANS L'APPROCHE DE LA PROXIMITE
}

\section{POLITICAL DIMENSION IN THE PROXIMITY APPROACH}

\author{
Juillet 2010 \\ Version révisée \\ Damien TALBOT \\ GREThA (UMR CNRS 5113) \\ Université de Bordeaux \\ Avenue Léon Duguit 33608 Pessac Cedex \\ Tél. : $0556848671 \quad$ Fax. : 0556848647 \\ damien.talbot@u-bordeaux4.fr \\ in \\ Géographie, Economie, Société, 2010, vol. 12, n², pp. 125-144.
}

\begin{abstract}
Résumé
L'opposition entre des acteurs hétérogènes, l'imposition d'arbitrages, le travail de mise en cohérence entre des intérêts a priori divergents sont des phénomènes sociaux qui conditionnent l'apparition des coordinations. Sans qu'ils soient ignorés ou niés, ces phénomènes d'ordre politique sont encore peu pris en compte dans une démarche proximiste qui privilégie une approche cognitive. L'objectif de ce papier est alors de proposer, au côté d'une vision cognitive, une lecture politique de la proximité. Pour ce faire, nous utilisons le concept d'institution, dont les liens avec la proximité sont explorés. Il apparaît que cette dernière est un fait institutionnel, qui se décline en proximités géographique et organisationnelle. La proximité géographique est ressource latente, seulement activée lorsqu'elle est articulée à une proximité organisationnelle. La proximité géographique vient renforcer ou limiter, selon qu'elle est souhaitée ou subie, une proximité organisationnelle qui consiste à coordonner cognitivement et politiquement les acteurs.
\end{abstract}

Mots clés : proximités, institutions, dimension cognitive, dimension politique.

\begin{abstract}
Opposing heterogeneous agents, imposing choices, and making divergent interests compatible, are all political prerequisites for agent coordination. Although these political prerequisites are not entirely ignored in proximity literature, such literature does tend to insist, for the most part, on cognitive perspective: the biggest beneficial effect of proximity is that of facilitating knowledge-sharing between agents. The principal objective of this paper is to propose both political and cognitive conceptions of proximity. In order to do so, we use the concept of institution, to examine the relations between these concepts - proximity and institution. We obtain one principal result: proximity is an institutional fact, which itself divides up two sub-categories, organizational and geographical proximity. Organizational proximity refers to the cognitive and political coordination of agents. Geographical proximity between actors is a latent resource. This resource would only be activated if the agents share organizational proximity.
\end{abstract}

Key words: proximities, institutions, cognitive dimension, political dimension. 


\section{LA DIMENSION POLITIQUE DANS L’APPROCHE DE LA PROXIMITE}

\section{Introduction}

Depuis maintenant une quinzaine d'années, l'approche par la proximité ${ }^{1}$ met en exergue le rôle des liens sociaux dans l'action économique, faisant l'hypothèse que la constitution de communautés autour de valeurs morales, de conventions, de normes, de croyances, de représentations, d'un lieu, etc., est un ingrédient essentiel pour atteindre un niveau suffisant de coordination d'une part, et affecte les modalités des coordinations d'autre part. L'endogénéisation du rôle de l'espace géographique dans la coordination en constitue l'apport le plus immédiat. Traditionnellement, l'espace avait été compris comme un cadre neutre et uniforme, sans relief autre que ceux identifiables par des coûts. L'espace était alors appréhendé d'abord par la notion de distance. C'est bien à un dépassement de cette vision restrictive que s'est attachée la démarche proximiste, pour prendre en compte les conséquences de la localisation de chaque acteur sur leurs modes de coordinations dans un espace hétérogène, asymétrique, multiforme, bref à chaque fois singulier et complexe (Gilly, Torre, 2000 ; Pecqueur, Zimmermann, 2004 ; Carrincazeaux, Grossetti, Talbot, 2008). Et puisque il est possible d'être présent « ici » et « ailleurs », autrement dit d'interagir à distance, la proximité s'est vue dotée d'une dimension non spatiale. Cette introduction élargit considérablement le champ d'analyse puisqu'il devient possible de questionner l'ensemble des modèles de coordination, que les acteurs soient géographiquement proches ou non. La conséquence est essentielle : l'analyse ne débute plus obligatoirement par l'étude des modes de coordinations déjà fondés sur une proximité géographique, mais elle peut y aboutir en expliquant les processus d'élaboration (Bellet, Colletis, Lung, 1993 ; Talbot, Kirat, 2005 ; Bouba-Olga, Carrincazeaux, Coris, 2008). Le territoire par exemple, comme catégorie de recouvrement des proximités spatiales et non spatiales n'est plus postulé, il devient un construit. Ainsi, le rôle de l'espace n'est ni ignoré, ni postulé.

Il s'agit là d'un acquis essentiel de la démarche proximiste. Il a été obtenu par l'introduction des résultats de divers courants théoriques, tout particulièrement ceux proposés par les sciences cognitives, puisqu'il s'agit avant tout de traiter des problèmes de coordination interindividuelle et collective. Le recours aux sciences cognitives s'est donc imposé de lui-

\footnotetext{
${ }^{1}$ Pour une présentation du groupe et des problématiques qui y sont développées, $c f$. Carrincazeaux, Lung, Vicente (2008).
} 
même, là comme ailleurs, pour mettre en lumière les conditions à la formation des coordinations collectives - dimension collective à laquelle se limite le présent papier.

Mais cette lecture cognitiviste n'épuise pas complètement la question. Sans qu'ils soient ignorés ou niés, l'opposition entre des acteurs hétérogènes dans leurs dotations en ressources, l'imposition de choix des uns sur les autres, le travail de mise en cohérence entre des intérêts a priori divergents sont des phénomènes sociaux qui président autant que les phénomènes cognitifs de convergence des représentations, de partage d'un langage commun, de valeurs etc., à la construction des coordinations collectives. Celles-ci, parce qu'elles sont tout autant des échanges que des épreuves de force, sont le résultat d'un travail cognitif et politique. Bien sûr, la distinction analytique entre les phénomènes sociaux qui relèvent du «cognitif » et du « politique » présente un côté caricatural, tant les faits empiriques relèvent simultanément des deux dimensions. Toutefois, cette distinction analytique présente le mérite, comme du reste la distinction entre dimensions non-spatiale et spatiale de la proximité, de souligner des phénomènes parfois sous-estimés, le politique dans un cas, le rôle de l'espace géographique dans l'autre. C'est finalement l'objet de ce travail que d'introduire la dimension politique dans l'approche proximiste.

Notre propos s'organise en trois temps. Nous montrons dans une première partie que, si l'économie de proximité est une approche d'abord cognitive, les questions d'ordre politique y sont très présentes. Puis, dans une deuxième partie, nous proposons d'introduire la dimension politique dans la démarche proximiste en mobilisant le concept d'institution, dont les liens avec le concept de proximité seront explorés. Les conséquences de cette introduction sur les définitions des dimensions de la proximité sont étudiées dans une troisième partie. Des exemples issus principalement de l'industrie aéronautique viendront étayer notre propos.

\section{La proximité, une approche d'abord cognitive ouverte à la dimension politique}

Les sciences cognitives ${ }^{2}$, qui d'une façon générale s'intéressent aux processus d'acquisition et d'utilisation des connaissances, constituent un gisement de savoir pour définir les fondements de l'action rationnelle, les procédures de la décision, pour aider à la compréhension des notions de règles, de conventions et tout particulièrement de représentations. L'apport des travaux cognitivistes à la proximité est indéniable.

\footnotetext{
${ }^{2}$ Qui regroupent de façon non exhaustive la psychologie, la linguistique, l'informatique, les neurosciences, etc.
} 


\subsection{La dimension cognitive dans la démarche proximiste}

Nous savons que chacun utilise des modèles mentaux, des représentations subjectives d'une entité concrète ou d'un état du monde pour agir. Ces représentations sont une réponse à l'incertitude qui envahit le monde de l'action : l'acteur se construit une image de la réalité, donne une signification aux données rassemblées, pour surmonter cette incertitude. Ce processus cognitif va lui permettre de continuer à agir, notamment collectivement. Par conséquent, le partage de représentations, qui ont pour première fonction cognitive de comprendre et d'expliquer la réalité, est une condition à toute coordination - interindividuelle ou collective. Dit autrement, une proximité dans les représentations donnant un même sens à une situation ou à un objet est donc nécessaire à la coordination.

De façon probablement plus indirecte, l'influence des sciences cognitives sur la démarche proximiste prend corps via l'interactionnisme qui place au centre de l'analyse une représentation particulière: celle, pour un acteur, des conséquences de ses actions. La cohérence de l'interactionnisme, qui renvoie dans les faits à une grande variété de travaux, se dessine autour du point de vue suivant: la société est construction permanente car elle n'existe qu'à travers les interactions entre les individus. Cette approche conçoit cette société comme une composition des relations interindividuelles et prend pour unité de base l'interaction, conçue comme un «champ mutuel d'influence » (Le Breton, 2004, p. 51). Sans voir ses comportements strictement déterminés par des éléments extérieurs, l'individu n'est pas non plus une monade détachée de toute influence. Le point clé est que l'individu se représente les conséquences de ses actions, fait de même avec celles des autres et agit. La réciprocité des perspectives est alors une condition au fait d'agir socialement: l'individu, dans son comportement, s'impose de prévoir comment les autres vont réagir. Inversement, lorsque les autres agissent à son propos, il connaît la gamme de comportements que ceux-ci attendent en retour.

La démarche proximiste a profité et profite encore heureusement de ces résultats, comme le montrent quelques travaux récents. De façon générale, toute définition de la proximité donne une place centrale aux interactions entre acteurs ou entre acteurs et objets, déplaçant la focale de l'individu vers l'interaction - qui devient de facto la nouvelle unité d'analyse ${ }^{3}$ - et sur la nécessité de l'existence d'un cadre cognitif commun. Chaque auteur proximiste intègre les apports des travaux cognitivistes. De manière non exhaustive, il est possible de citer les

\footnotetext{
${ }^{3}$ L'article de Bourdeau-Lepage et Huriot (2009) qui examine les liens entre proximité et interaction et reformule les formes de proximité en termes de coûts d'interaction illustre ce point.
} 
travaux de Kirat et Lung (1995) qui comprennent la proximité institutionnelle comme la capacité des acteurs à se coordonner par la convergence de leurs représentations vers un ensemble de règles partagées. Plus récemment, la proximité organisée se définit par la capacité qu'offre une organisation de faire interagir ses membres (Torre, Rallet, 2005). Cette capacité résulte à la fois d'une logique d'appartenance - à une organisation - et d'une logique de similitude - entre des représentations. La logique d'appartenance traduit le fait que les membres d'une organisation interagissent effectivement grâce et dans un même cadre de règles et de routines de comportements dont ils partagent les mêmes interprétations ; la logique de similitude exprime la communauté de croyances et de savoirs que partagent et qui lient les participants. Alors les coopérations entre les individus se développent d'autant mieux qu'ils appartiennent à la même firme ou au même réseau d'innovation. Bouba-Olga et Grossetti (2008) évoquent quant à eux une proximité de ressources dont sont dotés les individus et qui renvoient au partage de ressources matérielles et cognitives. Dans ce dernier cas, les auteurs définissent la proximité cognitive comme une complémentarité ou une similarité de valeurs, d'allant de soi, de routines, de références, etc. La proximité cognitive, comprise comme le partage d'une même base de connaissances, est alors considérée comme la condition essentielle à l'innovation car elle permet les apprentissages entre les agents (Boschma, 2005). Suire et Vicente (2008) proposent d'interroger la thèse selon laquelle les clusters seraient des lieux facilitant les innovations car la proximité géographique entre firmes innovantes serait un gage d'une meilleure exploitation des externalités de connaissances. Ils mettent en lumière la nécessité de maintenir un minimum de distance cognitive entre les partenaires, indispensable à des processus d'innovation fondés plus sur une complémentarité qu'une similarité des connaissances. Rychen et Zimmermann (2008), s'attachant à comprendre la structuration des clusters, développent la notion de knowledge gatekeepers pour expliquer le rôle tenu par un petit nombre d'agents qui font les liens entre les connaissances localisées au sein du cluster et les connaissances globales.

Toutes ces approches ont en commun de s'inscrire dans une perspective interactionniste qui conduit à voir dans la proximité un lien social de nature principalement cognitif, c'est-à-dire focalisant l'attention du chercheur sur les questions de traitement de l'information, de représentations, de production et de transfert de connaissances. Dans ces travaux, les questions plus politiques sont, non pas ignorées, mais seulement suggérées. Par exemple, Suire et Vicente (2008) concluent que, face au risque lié à la propriété intellectuelle que courent les firmes qui ouvrent leur base de connaissances en adoptant des stratégies 
d'accessibilité réciproque, la proximité géographique peut être utile entre les partenaires pour des raisons cette fois non cognitives. Les agents, insérées qu'ils sont dans des réseaux sociaux localisés, ont plus à perdre à adopter une stratégie opportuniste : il est parfois plus facile de tromper un inconnu que son voisin avec lequel on partage un même réseau d'amis, d'anciens étudiants, de parents d'élèves, etc. C'est ici la dimension du contrôle social qu'exerce un réseau sur le comportement de ses membres qui est suggérée. Et comme les réseaux sociaux ont tendance à être localisés dans un même lieu (Bouba-Olga, Grossetti, 2008), la proximité géographique viendrait en quelque sorte verrouiller la relation entre des agents qui auraient à payer dans leur relation personnelle des comportements opportunistes. Par ailleurs, le caractère éminemment stratégique contenu dans le rôle de knowledge gatekeepers, entendus comme des passeurs de connaissances, mérite d'être exploré : ces agents situés sont en capacité de filtrer les connaissances en fonction de leurs intérêts et plus généralement à contrôler les connaissances qui entrent ou sortent d'un cluster. Les phénomènes de lock in, engendrés d'une part par une distance cognitive trop faible entre des agents porteurs de connaissances trop similaires affaiblis dans leur capacité d'apprentissage et d'autre part par le poids des routines qui réduisent la capacité des mêmes agents à percevoir l'intérêt des nouvelles technologies (Boschma, 2005), peuvent ou doivent aussi s'interpréter comme une domination de certains acteurs en situation de rente qui réalisent des arbitrages dans le sens de leurs intérêts propres, même au risque d'enfermer un territoire dans une trajectoire sans issue.

Ce sont encore des pistes de recherche qui sont ici finalement ouvertes. Elles montrent qu'il ne faut pas réduire les phénomènes de proximité à des liens sociaux apaisés et sans conflits, convergeant quasi naturellement vers une vision partagée du monde, à la poursuite d'objectifs communs acceptés et rarement remis en cause. Hors, et le constat empirique en est permanent, les phénomènes de proximité, comme tous liens sociaux, incorporent des conflits d'intérêts, des remises en causes des objectifs, des affrontements quant au sens de l'action collective. Si l'action collective repose sur un travail cognitif de rapprochement des représentations, elle suppose pour exister un travail politique de mise en convergence des intérêts qui seront plus ou moins satisfaits par l'atteinte d'objectifs communs, d'arbitrages entre des intérêts inconciliables, d'imposition de choix qui apparaissent aux acteurs comme finalement légitimes, etc. Bref, les acteurs, pour se coordonner, s'opposent autant qu'ils s'associent.

Ce constat n'est pas nouveau : d'ores et déjà, des travaux sur les conflits d'usages de l'espace (Kirat, Torre, 2008) ou sur les dimensions indésirables de la proximité organisée (Torre, Zuindeau, 2009) ont été accomplis, démontrant que la dimension politique n'est ni absente et 
ni considérée comme étant hors de propos par les chercheurs du groupe «Dynamiques de Proximité ». D'ailleurs, affirmer que la localisation dans l'espace géographique et le positionnement dans un faisceau d'interactions conditionnent les activités économiques, productives, commerciales, d'innovation, etc. des acteurs (Pecqueur, Zimmermann, 2004) est une façon de reconnaître que la place dans une structure hiérarchique compte, que les rapports d'autorité sont aussi des rapports de proximité. C'est dans cette voie de recherche que s'inscrit ce papier, qui consiste à poursuivre le travail d'intégration dans la démarche proximiste de la dimension politique.

\subsection{La dimension politique : de quoi parle-t-on?}

Comment se résolvent les conflits inhérents à toute organisation, exemple paradigmatique du déploiement d'une proximité organisée? Comment se construisent les compromis que suppose l'action collective? Comment les problèmes sont-ils hiérarchisés ? Comment se construisent les choix collectifs, par exemple concernant la répartition des richesses? Comment s'imposent les décisions collectives ? Comment les acteurs font-ils prévaloir leurs intérêts ? Les questions posées ici sont d'ordre fondamentalement politique ${ }^{4}$ dans le sens où les réponses apportées par les acteurs font appel à l'exercice d'un pouvoir, à des relations d'autorité et d'obéissance. Ces questions renvoient de façon générale à la façon dont les acteurs mobilisent leurs ressources pour orienter, en exerçant une autorité légitime, les choix collectifs et satisfaire ainsi leurs intérêts. Dit autrement, l'affirmation de la dimension politique peut consister à poser la question des visées d'acteurs aux positions sociales différenciées dans le cadre d'une démarche proximiste ainsi «dénaturalisée », à mettre en exergue le «travail politique » réalisé par acteurs pour arriver à faire évoluer ou à stabiliser l'ordre en place. De manière non exhaustive, plusieurs types d'analyses s'inscrivant dans cette thématique se côtoient: une perspective économique institutionnaliste qui voit dans les questions politiques d'abord une question de régulation de l'action collective, la sociologie politique qui introduit justement l'idée de travail politique des acteurs, reprise par la sociologie économique.

Amable et Palombarini (2005) expliquent que dans une société hétérogène il est impossible d'agréger les attentes sociales pour fixer un unique objectif pour l'ensemble de la société. La

\footnotetext{
${ }^{4}$ Selon Braud (2008, p. 16), «le politique renvoie à ce champ social dominé par des conflits d'intérêts régulés par un pouvoir lui-même monopolisateur de la coercition légitime ». Chez Lagroye, François et Sawicki, (2006), l'espace politique est un domaine d'activité spécialisé qui se signale par la prétention qu'ont certains acteurs d'user d'un droit de commander les membres d'un groupe social.
} 
régulation du conflit social suppose que certaines attentes sociales seront négligées, sacrifiées. Certains acteurs ont alors intérêt à la déstabilisation du mode de régulation en place, puisque le cadre institutionnel existant ne prend pas en compte leurs attentes. Et d'autres verront dans cette crise une menace pour leurs intérêts. Le travail politique devient une tentative des acteurs de faire prévaloir par la coordination une organisation sociale conforme à leurs intérêts. Ils essayent par là même d'obtenir et de mettre en œuvre une capacité d'influence sur les choix publics, par exemple en définissant les priorités (Amable, Palombarini, 2004). La viabilité d'une action collective dépend donc de sa capacité à réguler les conflits, conflits qui ne peuvent être abolis. L'introduction de la dimension politique vise alors à expliquer la stabilité et la reproduction d'un système social régulé, malgré et en partie grâce aux contradictions dont il est rempli. C'est d'ailleurs dans cette perspective que travaille Commons $(1931,1934)$, pour qui les questions centrales des sciences sociales peuvent se résumer ainsi : pourquoi les interactions sociales, alors que les individus sont indépendants et en conflit, se réalisent-elles dans un contexte d'ordre et non de chaos? Comment se construit la sécurité des anticipations vitales aux coordinations ? Il remet en cause l'idée smithienne selon laquelle le marché est la source de l'ordre en assurant la coordination optimale des actions individuelles, car autorégulé par le système de prix. L'ordre ne s'explique pas uniquement par les seuls principes de système de prix et de liberté individuelle, pas plus qu'il n'est le résultat naturel d'une harmonisation inconsciente des intérêts du fait d'une main invisible. Au contraire, il est le résultat d'une régulation des interactions et des conflits par les règles (Bazzoli, 1999).

En sociologie politique, le travail politique en particulier vise à faire tenir ensemble ce que les logiques d'action séparent. Il se définit comme "l'art de s'arranger avec autrui » (Dubet, 2009, pp. 298-299). Une première illustration peut en être donnée avec le travail de légitimation. Certains individus et groupes accumulent des ressources du pouvoir - argent, prestige social, légitimité - et travaillent politiquement au maintien de ces répartitions inégales, tandis que d'autres contestent ces inégalités à leur propre avantage. Le travail de légitimation peut consister à fonder la légitimité de ces inégalités en faisant appel à un «tiers absent » (Dubet, 2009, p. 305) : la société, l'intérêt général, la justice, la démocratie, la mondialisation, les forces du marché, etc. Une seconde illustration possible concerne le travail politisation. La politisation consiste en une requalification des activités sociales (Lagroye, 2003). La diversification des pratiques et des rôles sociaux est à l'origine d'une sectorisation des espaces d'activités : il existe des activités pour éduquer, pour protéger l'environnement et 
la santé, pour être un citoyen, pour travailler, pour échanger des biens et des services avec profit, etc. La politisation vise à transgresser volontairement cette diversification, à brouiller les séparations instituées, pour requalifier un objectif d'un domaine - économique - en un autre - environnemental, sanitaire, etc. - et ce malgré l'absence de liens de causalité entre les objectifs, voire les contradictions qui peuvent exister entre ces derniers. Ainsi, une compagnie pétrolière déclare agir pour la protection de l'environnement, un groupe agro-alimentaire veut protéger la santé des consommateurs, un avionneur se pose en garant de l'indépendance nationale. Cette requalification peut aussi prendre la forme d' «une politisation d'un enjeu» (ibid., p. 367), ce qui consiste à inscrire en le reformulant un problème économique, social, culturel, technique, etc. dans la liste des questions traitées par des organisations explicitement politiques. Les objectifs assignés à l'action sont requalifiés, d'économiques, de sociaux, de culturels ou de techniques en politiques : ils en reçoivent un surcroît de légitimité aux yeux de tous. Plus Airbus vend d'avions, plus l'Europe se construit et plus il est utile pour tous qu'Airbus vendent des avions : ce qui est bon pour Airbus est bon pour l'Europe, à tel point que les dirigeants politiques se voient transformés en promoteurs des produits. On peut étendre l'illustration à l'industrie du nucléaire, de l'armement, du spatial.

Cette notion de travail politique des acteurs est reprise à son compte par la sociologie économique. Fligstein (1996) voit dans l'introduction du travail politique des acteurs une façon de faire référence à leurs stratégies et à leurs jeux de pouvoir, tout en permettant d'aborder le rôle de l'Etat et des collectivités publiques. Ce travail politique consiste en des stratégies qui ont vocation à maintenir ou contester l'ordre établi, faisant évoluer les institutions en place. N. Fligstein s'intéresse tout particulièrement au travail politique des firmes qui cherchent, soit à intégrer un secteur en cherchant à déstabiliser les barrières à l'entrée érigées par les firmes dominantes en place en exerçant un travail de lobbying sur l'Etat, soit parce qu'elles sont dominées dans leur propre secteur à empêcher les firmes dominantes de reproduire la hiérarchie existante. Inévitablement apparaissent des conflits entre les firmes auxquels l'Etat coercitif peut mettre fin.

A la croisée des travaux de J. Lagroye, de N. Fligstein et du courant régulationniste, Jullien et Smith (2008) voient dans la stabilité des règles qui orientent les firmes et les industries, audelà des nécessités fonctionnelles de conception, de production et de commercialisation des biens, le résultat d'une construction politique. "It follows that the critical condition for the stable regulation of an industry is the capacity of certain actors to impose and maintain a division of authority that rests upon justifications which are generally accepted by 
practitioners concerned»(ibid. p. 3). Le travail politique prend par exemple la forme d'alliances entre acteurs d'une même industrie - l'industrie aéronautique est sans conteste un exemple typique - pour peser sur les négociations auprès des autorités publiques locales, nationales ou internationales. Pour ce faire, un argumentaire allant dans le sens de l'intérêt général - sécurité, indépendance nationale, préservation de l'emploi, maintien de la croissance, etc. -, du respect de la légalité - normes environnementales ou sanitaires - ou de contraintes économiques - taux de change, coût du travail - est collectivement développé pour préserver au mieux les intérêts privés des alliés de circonstance. Les objectifs du groupe d'intérêt sont, par cet argumentaire, transformés en problèmes collectifs et publics, donc politisés ${ }^{5}$.

On comprend mieux, au vue de ces travaux, la nécessité d'affirmer la dimension politique dans une démarche qui vise à définir le cadre et les conditions qui rendent possible la coordination, notamment collective. Reste à réaliser cette intégration. Pour ce faire, nous proposons d'utiliser le concept d'institution car il permet, non pas de substituer une dimension - politique - à l'autre - cognitive -, mais d'enrichir la démarche proximiste par une prise en compte simultanée des phénomènes cognitifs et politiques. La partie suivante est alors consacrée à l'étude des liens entre les deux concepts, institution et proximité, pour démontrer l'intérêt d'un rapprochement.

\section{Institution et proximité}

Afin de réaliser un tel rapprochement, il est utile au préalable de revenir sur la définition de l'institution que nous mobilisons ici, tant ce terme semble renvoyer à des acceptions différentes - parfois même au sein des approches institutionnalistes.

\subsection{Bref retour sur l'institution}

Prenant des formes variées, toutes les institutions présentent la particularité de lier pensée et action. Il s'agit de connecter le monde mental et le monde extérieur concret et de reconnaître avec Mauss (1950) qu'il n'est pas possible de faire abstraction du contexte pour spécifier les pensées. Selon M. Mauss, les représentations collectives n'ont d'existence que dans la mesure où elles commandent des actes. L'action devient une sorte de langage, traduisant une idée, la

\footnotetext{
${ }^{5}$ Et Jullien et Smith (2008) d'ajouter qu'ils peuvent être dépolitisés lorsque les arguments utilisés font appel à l'expertise ou l'efficacité.
} 
croyance se manifestant dans la conduite. En sociologie politique, toute institution se présente d'abord comme un ensemble de pratiques, de tâches particulières, de règles de conduite entre des personnes, tout en étant aussi les croyances et les représentations qui concernent ces pratiques et qui tendent à justifier leur existence (Lagroye, François, Sawicki, 2006). De même, du côté de la théorie néo-institutionnelle sociologique, W.R. Scott lie monde mental et monde concret en définissant les institutions «as social structures that have attained a high degree of resilience, [...] composed of cultured-cognitive, normative, and regulative elements that, together with associated activities and resources, provide stability and meaning to social life » (Scott, 2001, p. 48). Les institutions sont des structures sociales durables, faites d'éléments symboliques, d'activités sociales et de ressources matérielles. Tenant compte de la dualité pensée / action, l'institution est à la fois la règle et le comportement, la représentation et les pratiques, les habitudes de pensée et de faire, sans que les uns ne soient réductibles aux autres. Il s'agit en quelque sorte de « faire » des idées collectives ${ }^{6}$. L'institution devient alors une idée commune en acte (Talbot, 2008a).

Telles quelles, les institutions ne sont pas directement observables. Cependant elles trahissent indirectement leur existence en prenant des formes variées. Ainsi, les règles sont souvent perçues comme la forme la plus apparente et la plus achevée de l'institution et y sont couramment assimilées. Plus généralement, les usages informels - coutumes, code moral, etc. - sont des institutions qui sanctionnent et imposent des tabous. Il en est de même de toutes les organisations formelles - le gouvernement, l'université, l'Eglise, etc.- qui exercent une autorité coercitive sur leurs membres. Dans un groupe social, chacun se doit d'adopter des pratiques habituelles, considérées comme «normales » par les autres membres. Les pratiques sont alors instituées. C'est le cas des rôles : être dans son rôle consiste à agir en fonction de la position qu'on occupe dans l'organisation, à l'instar d'un magistrat, d'un enseignantchercheur, d'un chef de projet, d'un maire, etc. Sont aussi institués des dispositifs matériels et des lieux, comme les prétoires, les salles de cours, les sites d'assemblage d'une firme architecte-intégrateur, les villes.

\subsection{La proximité, un fait institutionnel}

La proximité peut être interprétée comme un fait institutionnel. Cette caractéristique se fait jour si l'on distingue proximité et distance.

\footnotetext{
${ }^{6}$ Searle $(1998,2005)$ utilise l'expression de «faits mentaux » pour qualifier les faits institutionnels.
} 
Pourquoi la proximité n'est pas l'inverse de la distance ? En quoi se distingue-t-elle de la distance ? Les travaux de J. Searle peuvent contribuer à établir une telle distinction. Selon Searle $(1998,2005)$, les faits peuvent être de deux ordres. Les faits bruts - Paris est à l'ouest de Bruxelles - existent, que nous le croyons ou non. Les faits peuvent aussi être mentaux - j'ai mal. Au sein de ces derniers ${ }^{7}$, il relève qu'il y a des choses, comme la monnaie, les propriétés foncières, les gouvernements, qui n'existent que parce que nous y croyons. Ce sont des faits institutionnels qui, s'ils ont l'apparence de l'objectivité, sont dépendants d'un accord humain. Une rangée de pierres fait office de frontière, parce que nous y croyons et parce que nous le voulons collectivement. Un bout de métal devient une pièce de monnaie uniquement parce que nous le décidons et l'acceptons collectivement. Ajoutons que cet accord collectif n'a rien de spontané : il est le résultat d'un travail politique de régulation des conflits, d'imposition d'intérêts, d'arbitrages considérés comme légitimes. Cet accord exprime un rapport de force à un moment donné entre des parties prenantes aux positions sociales et aux ressources inégales. L'accord collectif constitue la première caractéristique d'un fait institutionnel.

Mais sur quoi porte cet accord collectif ? Sur la fonction assignée à la rangée de pierres ou au bout de métal. Ces faits institutionnels renvoient à des faits bruts auxquels ils sont irréductibles. Ces faits bruts sont transformés en faits institutionnels par l'imposition collective d'une fonction. C'est la seconde caractéristique d'un fait institutionnel. La fonction n'est pas intrinsèque à la physique du phénomène, à sa nature, mais assignée de l'extérieur par des utilisateurs conscients et relativement à leurs intérêts. En d'autres termes, l'assignation de fonction est toujours relative à l'observateur. Les fonctions que nous attribuons aux faits bruts sont des construits institutionnels. Finalement, cette assignation collective crée un nouveau fait, qualifié d'institutionnel car il besoin des institutions, d'idées communes en actes, pour exister.

Nous proposons d'utiliser la distinction fait brut $v s$ fait institutionnel pour différencier distance et proximité et par là-même expliquer en quoi la proximité est un fait institutionnel. La distance physique entre des objets et/ou des individus est un fait brut. La proximité quant à elle peut se définir comme un fait institutionnel non réductible à l'inverse d'une distance brute. La volonté commune d'être proches et l'assignation collective d'une fonction à la proximité la distingue du fait brut qu'est la distance. Reprenons ces points.

\footnotetext{
${ }^{7}$ Searle (1998) présente une taxonomie des faits mentaux qui déborde notre propos. Cf. p. 159 pour une présentation exhaustive de celle-ci.
} 
Nous sommes proches, au sens institutionnel du terme, tout d'abord parce que nous le voulons collectivement. La proximité n'est pas un fait objectif, même si nous la parons parfois d'une certaine évidence, d'une certaine naturalité : il semble évident, naturel, aux yeux de tous que les habitants d'une même ville soient proches et partagent quelques valeurs. Or, s'il est incontestable qu'ils sont proches physiquement, rien n'indique a priori qu'ils le soient institutionnellement. On peut être concrètement à faible distance physique de quelqu'un, parce que les circonstances nous conduisent à une co-localisation par exemple avec les habitants de sa ville: le fait brut est incontestable, mais il ne résulte pas d'un accord des parties autre que de l'inévitable acceptation des contraintes d'entassement dans l'espace. On peut être physiquement proche de son voisin de pallier sans pour autant décider d'être proche institutionnellement, ce qui nous permet d'ailleurs de nous ignorer l'un l'autre.

Comme produit institutionnel, la proximité se voit attribuer par les acteurs qui se reconnaissent proches une fonction de création d'un potentiel relationnel. Elle doit faciliter les éventuelles coordinations collectives. Un effet non désiré est bien sûr les effets négatifs de la proximité - $c f$. infra. Il s'agit là d'une fonction non souhaitée qui échappe à quelques agents, ce qui montre que l'assignation de fonction est un rapport de force.

Deux autres fonctions complémentaires sont assignées collectivement à la proximité : délimiter les frontières spatiales et sociales d'un groupe d'individus et ainsi contribuer à différencier les groupes les uns des autres. La proximité crée un «dedans » et un « dehors » en différenciant les groupes sociaux. Se sentir proche, c'est en même temps se sentir éloigné d'autres acteurs. Si la proximité a pour fonction d'inclure dans le groupe des acteurs qui possèdent les caractéristiques requises - localisation, ressources matérielles, représentations, maîtrise d'un langage, acceptation de valeurs, capacité à tenir son rôle - elle exclut en même temps ceux qui ne disposent pas des caractéristiques idoines.

Il faut à ce stade souligner que si la proximité est irréductible à l'inverse de la distance, la proximité ne peut exister sans une faible distance - spatiale ou non. Autrement dit, il n'y a pas de faits institutionnels sans faits bruts. Searle (1998) nous rappelle cette évidence : là où il y a une fonction assignée à quelque chose, il doit y avoir quelque chose à quoi elle est imposée. La faible distance est en quelque sorte le support brut sur lequel on impose intentionnellement les fonctions de la proximité. La première ne fait pas appel à la volonté des acteurs, tandis que la seconde est acceptée par conviction, par intérêt ou encore par soumission. L’idée semble assez simple à saisir lorsqu'on se réfère à la distance spatiale : la proximité se construit ici sur une faible distance physique, qui existe, que nous y croyons ou non, que nous l'acceptons ou 
non et qui se voit attribuer des fonctions. Elle se complique quelque peu lorsqu'on renvoie au fait brut de la distance sociale. En quoi ce fait est-il brut ${ }^{8}$ ? Par distance sociale il est fait ici référence aux attributs des acteurs en termes de ressources cognitives et matérielles, leur âge, leur santé, leur sexe, etc., bref l'ensemble des critères qui permettent de classer les individus en groupes plus ou moins homogènes. La distance sociale concerne aussi des acteurs collectifs : pensons aux firmes d'un même secteur partageant les mêmes marchés, les mêmes technologies, les mêmes produits, les mêmes fournisseurs, les mêmes standards et autres cadres législatifs.

Selon qu'elles se construisent sur de faibles distances spatiale ou sociale, il est possible de distinguer deux grandes catégories de proximités. Il y aurait d'un côté un fait institutionnel, une proximité géographique, existant par un fait brut, une faible distance spatiale. De l'autre côté, une proximité organisationnelle, existant grâce à une faible distance sociale.

\section{Les dimensions de la proximité}

Observons les conséquences de l'introduction de la dimension politique, via l'institution, sur les définitions des dimensions de la proximité. Pour ce faire, nous conservons la distinction séminale entre deux catégories de proximité. D'une part, on peut bien sûr être proche géographiquement. D'autre part, on peut être «proche» tout en étant éloigné géographiquement: la proximité, fait institutionnel, présente au côté de la dimension géographique une dimension de type organisationnel.

\subsection{La proximité géographique}

La proximité géographique peut prendre deux valeurs fondamentales : les acteurs se croient « être proche de » ou « être loin de », ces deux valeurs constituant les extrémités d'un même continuum. Elle n'existe pas sans une faible distance physique entre des objets et/ou des individus, qui constitue le fait brut support de l'assignation des fonctions - auquel toutefois la proximité géographique ne se réduit pas. La faible distance physique devient une proximité géographique lorsque les acteurs lui assignent les fonctions de facilitateur des interactions en face en face et attribuent à l'espace un usage spécifique et un rôle de typification d'un groupe

\footnotetext{
${ }^{8}$ Cette faible distance sociale n'est pas un fait rigoureusement brut et relève déjà d'une construction sociale. Mais elle reste de l'ordre de la ressemblance de caractères entre acteurs, sans qu'aucune intention collective au sens de J. Searle n'ait été exprimée par ces mêmes acteurs.
} 
social. A l'instar de Torre et Caron (2005), nous distinguons le cas de la proximité géographique souhaitée de celui de la proximité géographique subie.

\subsubsection{Proximité géographique souhaitée}

Dans le cas d'une proximité géographique souhaitée, on constate l'existence d'un accord collectif sur les fonctions à lui attribuer, ce qui suppose un profond travail politique des acteurs pour atteindre cet accord. Les acteurs accordent collectivement à la proximité géographique une première fonction de facilitation des interactions en face à face. Nous renvoyons à l'idée classique selon laquelle l'espace physique est structuré par des infrastructures de transport et de communication. Il constitue alors un cadre matériel favorisant la circulation des informations, des biens physiques, des individus et facilitant les interactions en face à face. Ce type d'interaction est souvent recherché car il réduit l'incertitude issue de la dimension tacite inhérente à tous savoirs et les risques d'opportunisme (Boschma, 2005). Dans le cas d'une proximité géographique permanente, l'interaction en face en face devient possible à organiser dans un délai très bref. Le face à face peut aussi être obtenu par une proximité géographique temporaire. Tout au long du processus productif par exemple, le besoin de proximité géographique peut varier, être par exemple intense lors des phases communes de conception et de développement (Carrincazeaux, Lung, 1998), comme dans le cas des équipes plateaux réunissant pour quelques semaines donneurs et preneurs d'ordres sur un même site pour co-concevoir des composants d'une automobile ou d'un avion. La mobilité des individus est aussi une façon de permettre des interactions de face à face qui restent à certains moments essentiels à toute vie sociale en général, et aux activités productives en particulier. Le besoin de proximité géographique temporaire peut être satisfait dans divers espaces, comme l'entreprise - réunions, équipes plateaux -, ou les temporary clusters - foires, conférences (Torre, 2009). Donc peu importe le lieu : la fonction n'est pas associée à un espace particulier.

Un deuxième ensemble de fonctions est attribué à l'espace concernant son usage. L'espace est en effet structuré matériellement selon les usages qui en sont attendus : une zone résidentielle s'accompagne de services s'adressant à des personnes de différentes tranches d'âges dans un cadre subissant le moins de nuisances possibles, une zone d'activité économique devra proposer une offre hôtelière et des services de transports, une zone touristique doit s'inscrire dans un environnement sain tout en offrant toute une série d'activités ludiques et culturelles, etc. Les acteurs attribuent un rôle spécifique à chaque espace qu'ils utilisent : celui de faciliter 
la vie quotidienne, de permettre le travail, de s'instruire, de s'amuser, etc. La distribution des rôles résulte d'un choix éminemment politique, d'arbitrages entre des intérêts divergents à l'origine de conflits d'usage - cf. infra.

Les acteurs assignent collectivement une troisième fonction de localisation d'un groupe et d'attribution de valeurs, de coutumes, d'habitudes, bref de «typification ». L'espace constitue un référent cognitif qui ne doit pas uniquement s'entendre comme un contexte purement physique doté d'attributs matériels au sein duquel se déroulent des relations économiques. Tous les acteurs associent des valeurs, des représentations, des coutumes, des modes de vie, une histoire, un nom, des limites physiques et administratives, une mémoire des coordinations précédentes réussies ou échouées ${ }^{9}$, des conflits, etc., à une aire géographique. L'espace joue alors un rôle dans la démarche de typification, démarche qui consiste à identifier des personnes, des objets, des actions à des «types » généralisables (Lagroye, François, Sawicki, 2006), à proposer un système de classement (Braud, 2008), ici en fonction de leur localisation. Se réclamer d'un lieu revient donc à se réclamer d'un groupe social, par association. Par exemple, Toulouse est aujourd'hui encore associée aux pères fondateurs de l'industrie aéronautique française - Morane, Dewoitine, Latécoère, etc. -, reste la ville où sont effectués les premiers essais en vol des nouveaux programmes - Caravelle, Concorde, famille Airbus - et où sont assemblés la majorité des avions de la gamme Airbus. Même si cela est moins vrai aujourd'hui, les industriels toulousains, qu'ils soient donneurs ou preneurs d'ordres, ont utilisés cette image positive dans leurs relations productives. Le lieu intervient alors dans le processus de construction - ou de destruction - des identités, puisque les acteurs locaux le font exister au regard des autres (Laganier, Villalba et Zuindeau, 2004).

Cette fonction est spécifique à chaque lieu, varie en intensité d'une localisation à l'autre. Certaines firmes développent une stratégie de différenciation basée sur les spécificités du lieu de leur production, en promouvant par exemple des labels d'indications géographiques comme les Appellations d'Origine Contrôlée: elles tirent leur légitimité des valeurs l'authenticité, la qualité, etc. - associées à l'espace géographique. Au-delà de cette vision cognitive, la fonction de typification induit une séparation entre les acteurs selon leur placement dans l'espace. Une façon de délimiter le groupe social consiste à délimiter «son » espace par des frontières. La fixation des frontières est le résultat d'un processus politique de

\footnotetext{
${ }^{9}$ A ce sujet, Colletis et Pecqueur (2005) dotent les territoires d'un patrimoine constitué par la mémoire des coordinations réussies et la confiance qui en résulte, et par des ressources cognitives spécifiques virtuellement complémentaires. La proximité se définirait alors comme l'attribut d'une personne concernant son accès à un patrimoine.
} 
régulation de conflits, de négociation, de passage de compromis, d'un travail politique aboutissant à des « arrangements spatiaux » (selon les termes de Lussault, 2007) que sont par exemple les Pays, les Régions, les communes, etc.

\subsubsection{Proximité géographique subie}

La proximité géographique subie renvoie au cas où les acteurs sont en conflits sur les fonctions à attribuer à une faible distance physique, dont ils subissent la réalité du fait brut. D'un point de vue politique, un rapport de force défavorable peut même conduire des acteurs à se voir imposer des fonctions. Comme la création de faits institutionnels revient à imposer une fonction à un objet, une personne, un évènement qui en était dépourvue, Searle (2005) considère que cette création impose un pouvoir, générateur de droits (capacité à) et d'obligations (contraint de). Ce n'est pas la pièce de monnaie comme objet qui compte, mais plutôt le fait que le détenteur de la pièce a désormais un pouvoir qu'il n'avait pas sans cela. Ce pouvoir, lorsqu'il est utilisé, est générateur de conflits soit parce que la fonction attendue n'est pas remplie, soit parce que différentes visions sur la fonction à attribuer à l'espace s'opposent. Il en est ainsi des conflits d'usage de l'espace, d'autant que les différents usages ou intentions d'usages de l'espace - usages résidentiels, productifs, récréatifs ou de préservation de la nature - sont d'autant moins compatibles qu'ils se déploient en un même lieu (Kirat, Torre, 2008). La proximité géographique se transforme dans ce cas en une contrainte pour le voisinage, tant la mobilité des acteurs pour se soustraire à un voisinage jugé indésirable peut être très coûteuse, voire impossible.

La superposition des usages peut d'ailleurs être révélatrice de conflits portant cette fois sur les représentations et les valeurs qui sont associées à un lieu. Le désaccord porte alors sur la typification de l'espace, par exemple entre des acteurs anciennement installés et de nouveaux arrivants. Mais, remarquent Kirat et Torre (2008), la mise en œuvre de projets, tout particulièrement ceux susceptibles de créer des nuisances, la rareté des sols disponibles, l'apparition progressive de dispositifs de gestion publique des sols à une échelle territoriale nouvelles règles d'urbanisme par exemple - sont aussi des facteurs d'expression ou d'émergence d'identités territoriales. Les conflits d'usage, en fédérant les usagers contestataires autour de valeurs communes, peuvent alors paradoxalement renforcer la typification d'un espace. 
Au final, l'existence d'une proximité géographique ne doit pas être interprétée comme l'assurance qu'une coordination collective s'instaure. Ce fait institutionnel ne repose encore sur aucune connexion positive des acteurs et n'implique pas une relation systématique, tout en lui ouvrant la voie. Cela signifie simplement que l'existence d'une forte proximité géographique entre acteurs n'est pas neutre : elle est une mise en disponibilité relationnelle, une ressource encore latente. La proximité géographique ne joue que dans son articulation à une proximité de nature organisationnelle, qu'elle soutient, renforce, compense, voire détruit en s'avérant être une source de conflits.

\subsection{La proximité organisationnelle}

Les acteurs partagent une proximité organisationnelle lorsque, d'une part, ils sont conscients d'avoir en commun des ressources cognitives comme les représentations, les valeurs, les coutumes, etc. - et, d'autre part, intègrent une hiérarchie en y prenant une place et en assumant le rôle afférant. Une telle proximité se déploie au sein des organisations comme les firmes, des réseaux d'innovation, des alliances stratégiques en vue par exemple d'effectuer un travail politique, des relations productives, etc. Elle se construit sur une faible distance sociale à laquelle les acteurs assignent des fonctions de coordinations cognitive et politique, transformant un fait brut - la faible distance sociale - en un fait institutionnel - la proximité organisationnelle.

La coordination cognitive consiste à acquérir, conserver et transmettre des connaissances, des représentations, des savoir-faire, des expériences, etc. La coordination politique implique d'exercer une autorité légitime en prenant des décisions par nature partiales tout en donnant une cohérence à des aspirations contradictoires. La première répond à la problématique de l'efficacité des actions, la seconde à la problématique de la conformité et de la légitimité des mêmes actions réalisées par des acteurs hétérogènes. Ainsi, dans la firme, la division interne du travail relève à la fois d'une nécessité cognitive de donner un contenu à des tâches, mais aussi d'une nécessité politique d'articuler les actions d'acteurs aux statuts et aux intérêts différents - actionnaires, direction, salariés, syndicats - et de répartir les richesses.

Le travail politique de contestation ou de maintien de l'ordre établi, comme toute coordination collective, se fonde pour s'exercer sur une proximité organisationnelle qui se déploie au sein 
des «groupes d'intérêt ${ }^{10}$ : ce travail politique appelle des alliances stratégiques entre catégories d'acteurs qui transforment une faible distance sociale - appartenir au secteur aéronautique - en une proximité organisationnelle - groupe d'intérêt - par l'octroi de fonctions de coordination. La politisation des objectifs des firmes, transformant des intérêts privés nombre d'avions vendus pour Airbus - en intérêts publics - construire l'Europe - nécessite un profond et permanent travail pour qualifier les intérêts et brouiller les séparations instituées entre activités industrielles et politiques. Ce travail de politisation s'accompagne d'un travail de légitimation. Le maintien de l'industrie aéronautique en tant qu'industrie de souveraineté, qu'il faut alors à ce titre publiquement financer, suppose un incessant travail d'explication auprès entre des Etats financeurs et des contribuables : l'aéronautique concoure au prestige de la Nation - le tiers absent chère à Dubet (2009) -, symbolisant sa grandeur et son indépendance ${ }^{11}$.

On le devine aisément dans les exemples précédents, la distinction entre dimension cognitive et dimension politique est purement analytique et renvoie à des découpages disciplinaires institués. Les faits institutionnels relèvent simultanément des deux dimensions et ne sont réductibles ni à l'une, ni à l'autre, les niveaux cognitif et politique étant au contraire profondément intégrés. Ainsi, coordinations cognitive et politique procèdent toutes deux d'une mise en cohérence des actions individuelles pour permettre une action collective. Notamment, les règles, en prévenant temporairement les conflits, empêchent ces derniers de ruiner la sécurité des anticipations nécessaire à l'action collective dans un monde incertain (Bazzoli, Kirat, 1996). Lorsqu'il s'agit de résoudre des conflits, la référence à des valeurs partagées et l'acceptation des règles générales de négociation conditionnent le démarrage du processus de négociation (Torre, Zuindeau, 2009). Eymard-Duvernay (2001) confère aux représentations une dimension politique, car elles intègrent à l'action individuelle la volonté de construire un monde commun avec les autres. Ces qualités permettent la sélection parmi l'ensemble des représentations des plus légitimes à fonder une société, un collectif. Fligstein (1996) fournit un bel exemple de cette articulation de travaux cognitif et politique lorsqu'il analyse la façon dont les firmes dominantes dans un secteur reproduisent les positions asymétriques en place. Les entreprises font appel pour effectuer le travail politique de reproduction de la hiérarchie sectorielle à une croyance partagée, dite «conception of

\footnotetext{
${ }^{10}$ Que Braud (2008) comprend comme des organisations qui cherchent à influencer les pouvoirs publiques dans un sens favorable aux insatisfactions qu'elle prend en charge. Pour une analyse du rôle des groupes d'intérêts dans l'action publique, $c f$. par exemple Hassenteufel (1995).

${ }^{11}$ Même si dans le cas français, il faille en passer par des coopérations européennes pour faire face aux coûts.
} 
control» (Fligstein, 1996, p. 658). Cette croyance porte sur la façon dont le secteur doit être structuré : ces représentations «disent» quelles sont les firmes dominantes et les firmes dominées. De façon plus générale, Braud (2008) souligne qu'il n'existe pas d'action collective viable sans que ne soient inculquées aux participants des représentations relatives au pouvoir, c'est-à-dire « des convictions communes concernant la nécessité des allégeances à la communauté et à la légitimité du gouvernement qui la régit» (ibid., p. 319). Elles encodent la distribution des pouvoirs, chacun se représentant les rapports de domination. Il apparaît alors que les représentations sont l'expression des intérêts des groupes dominants, qui imposent leurs visions du monde (Muller, 2005, 2008). La maîtrise des représentations du réel devient critique. Ces représentations valorisent une dimension d'un problème, en occultent d'autres, en fonction de la capacité des acteurs à imposer leurs points de vue grâce à un travail politique, notamment de qualification qui restreint l'espace du choix des solutions envisageables (Hassenteufel, 2010). Alors, selon P. Muller, «le référentiel constitue [...] une contrainte pour les acteurs, à la fois parce qu'il transforme leur perception du monde vécu et disqualifie les anciennes visions du monde » (ibid., 2005, p. 172). Ces travaux ont en commun de montrer qu'il existe alors des utilisations «stratégiques » des représentations qui peuvent contribuer au renforcement d'une position.

Par ailleurs, la coordination politique n'est possible que s'il existe une croyance partagée chez les participants, celle que chacun agira en fonction de son rôle et non en fonction de ses humeurs, de ses intérêts strictement personnels (Lagroye, François, Sawicki, 2006). Certaines relations, même si elles prennent la forme pacifique d'une négociation, deviennent des épreuves de forces qui se soldent par l'apparition d'un dominant et d'un dominé. Elles ne dégénèrent pas en violence car les partenaires appliquent des règles qu'ils ont appris à connaître et qu'ils respectent en pratique. Il en est ainsi des règles de droit dont la robustesse est issue de croyances concernant leur utilité pour écarter l'arbitraire, la violence, etc.

Enfin, intégrer une structure hiérarchique dans une organisation implique une prise de rôle qui passe par l'acquisition de savoirs pratiques permettant à chacun d'accomplir correctement ce qu'on attend de lui. Ainsi des routines sont attachées à des rôles dans le sens où ce sont des savoir-faire pratiques à accomplir lorsqu'on occupe une place précise dans une structure hiérarchique. L'acquisition de ces savoirs est bien le résultat d'un apprentissage : pour tenir sa place dans un collectif - se coordonner politiquement avec autrui - il faut apprendre des autres une série d'actes à accomplir - se coordonner cognitivement avec autrui. 


\subsection{Des proximités articulées}

Reste à articuler les proximités géographique et organisationnelle en dernière étape de la démarche proximiste. La proximité géographique, lorsqu'elle est souhaitée, est un potentiel relationnel que les acteurs peuvent mobiliser dans leur coordination collective : les fonctions de typification d'un groupe social associé à un lieu, d'affectation d'un usage et de facilitateur des interactions en face à face du fait d'un espace géographique structuré vont soutenir les coordinations cognitive et politique. En effet, le partage de valeurs, de représentations, d'une histoire associée à un territoire et les fréquents face à face vont générer un surplus de confiance entre les acteurs. Les coordinations collectives locales bénéficient en outre de l'encastrement des relations productives dans des réseaux sociaux localisés : les coopérations ont tendance à d'autant mieux s'établir entre des acteurs appartenant à des organisations différentes qu'ils sont issus de la même université ou Ecole, qu'ils appartiennent au même réseau familial et social (Grossetti, Bes, 2001).

Ce fut le cas dans les années soixante et soixante-dix entre donneurs et preneurs d'ordres toulousains participant au programme du supersonique Concorde. Donneurs et preneurs d'ordres locaux constituent à cette époque un « cercle fermé » dans lequel les mécanismes de concurrence jouaient plus faiblement qu'ailleurs du fait de l'existence d'une forte confiance et de l'insertion des relations productives dans des réseaux sociaux, ce qui rendrait très coûteux tout comportement opportuniste. Un réel travail politique de maintien de cet ordre établi fut réalisé par l'avionneur toulousain et les principaux fournisseurs localisés à proximité. Cet ordre fut contesté par Airbus dans les années quatre-vingts dix lorsque cet architecteintégrateur fit appel à des fournisseurs mieux-disant en termes de qualité, de délai et de prix mais plus forcement co-localisés. Plus récemment, la politique publique des pôles de compétitivité vise à accentuer l'agglomération des acteurs susceptibles d'innover en commun, en espérant que la proximité géographique, comme facilitateur d'interactions en face à face, jouera son rôle bénéfique de soutien à la proximité organisationnelle. Cette politique en soutenant les projets réunissant des grandes firmes, des PME et des laboratoires de recherche locaux, cherche à créer progressivement au fil des rencontres une mémoire des coordinations cognitives et politiques réussies et donc de la confiance entre des acteurs privés et publics. Elle vise aussi à s'assurer que les retombées de l'effort public de financement de la recherche seront locales, via le soutien aux PME régionales. Dans le cas du Pôle de compétitivité Aerospace Valley, réunissant les acteurs aquitains et midi-pyrénéens de l'aéronautique, du spatial et des systèmes embarqués, on constate que le principe de captation d'une très grande 
part des aides publiques par les avionneurs et les principaux systémiers est remis en cause : les PME régionales ont aujourd'hui accès à ce type de financement de la recherche et voient ainsi un long travail politique de contestation auprès des instances régionales couronné de succès. Ici la proximité géographique qui conditionne l'accès au financement a en outre permis l'intensification de ce travail politique, d'une part, en favorisant les face à face entre décideurs locaux, d'autre part, en raison du fait que ces PME contestataires appartiennent aux deux Régions administratives.

Parfois la proximité géographique permanente peut ne jouer aucun rôle dans les coordinations. Ainsi, un groupe multi-établissement compense la dispersion de ses sites par le développement d'une proximité organisationnelle intra-firme, à l'instar d'Airbus (Talbot, 2008b). Airbus SAS est le résultat de consolidations de l'industrie aéronautique européenne d'abord infranationales, puis internationales. La Société à Action Simplifiée hérite donc d'une grande quantité de sites dispersés en Europe, éclatement spatial avec lequel il composer pour concevoir, fabriquer et assembler un produit aussi complexe qu'un avion de ligne. Cette dispersion des sites de production est compensée de deux façons.

En premier lieu, une proximité géographique temporaire est crée grâce à la constitution d'équipes plateaux utiles lors des phases de conception d'un appareil - programmes A340500/600 et A380. Le face à face en effet, tout particulièrement dans les phases de conception commune, demeure essentiel pour répondre à des questions techniques et productives nouvelles, car il permet de fluidifier les relations et de rendre les discussions plus interactives, facilite grandement la recombinaison des compétences dispersées en permettant le transfert de connaissances (Rallet, Torre, 2007) et accentue les possibilités de contrôle du partenaire.

En second lieu, des outils de coordination cognitive et politique viennent compenser la dispersion spatiale, de la conception à l'assemblage de l'avion. Airbus doit surmonter deux obstacles concernant la coordination cognitive : gérer une multiplicité de sites dispersés géographiquement et porteurs de compétences qui se chevauchent, voire se dupliquent; favoriser la diffusion des routines entre des unités de cultures et de passés différents. Est créé un réseau interne de circulation d'information et des résultats de la recherche par l'utilisation des Technologies de l'Information et de la Communication. Plus qu'un simple échange de données, le système fonctionne comme une mémoire partagée constitutive d'une proximité organisationnelle fournissant autant de repères pour le travail en commun. La coordination politique se trouve quant à elle singulièrement renforcée par la création de la société intégrée Airbus SAS, accentuant le contrôle de la société mère européenne sur ses filiales allemandes, 
espagnoles et françaises puisque une direction unique est créée. Cette direction unique impose une redistribution des rôles aux sites spécialisés. Toutefois ce contrôle n'a pas été toujours suffisant. Les nombreux retards de mise en production de l'A380 résultent in fine d'un manque de contrôle politique du siège français d'Airbus sur ses sites allemands, auxquels la direction n'a pas su imposer l'utilisation d'un outil de conception de câblage des tronçons commun à l'ensemble du groupe, au profit d'un outil développé in situ qui n'a pas permis la connexion du tronçon allemand au reste de l'avion. Un défaut de coordination politique - une direction qui n'impose pas l'utilisation d'un outil commun - qui provoque une mauvaise coordination cognitive - des apprentissages entre sites franco-allemands insuffisants. Un défaut de proximité organisationnelle en somme.

\section{Conclusion}

Ce travail s'inscrit dans la lignée de travaux récents qui ont déjà soulignés que les relations de proximité incorporaient en leur sein des rapports sociaux de nature politique. Pour développer cet axe de recherche, nous avons procédé en deux temps. En premier lieu, nous avons donné un contenu à ce que nous nommons «dimension politique » en développant le concept de «travail politique» des acteurs, jusqu'alors peu ou pas étudié dans l'approche de la proximité. Il a permis de justifier de la nécessité d'une plus grande prise en compte de la dimension politique dans cette dernière. Pour ce faire et en second lieu, nous avons, en étudiant les liens analytiques entre institution et proximité, introduit la dimension politique dans la définition même de la proximité. Il est apparu que, comme fait institutionnel, la proximité se distingue de la faible distance dans le sens où les acteurs assignent collectivement à cette dernière des fonctions : dans le cas d'une proximité géographique, il s'agit de faciliter les interactions en face à face, d'attribuer un usage à l'espace et de typifier un groupe social ; dans le cas d'une proximité organisationnelle de coordonner cognitivement et politiquement les acteurs qui s'engagent dans une action collective. La proximité géographique, comme potentiel relationnel, vient renforcer ou limiter selon qu'elle est souhaitée ou subie la proximité organisationnelle.

Ces premiers résultats en appellent d'autres, par exemple concernant les liens entre proximité, politique et pouvoir. Dans ce travail, le pouvoir a joué son rôle de «banni récalcitrant » selon l'expression chère à Perroux (1973) : par exemple, vouloir introduire dans l'analyse la question du travail politique, c'est-à-dire celle du maintien ou de la contestation de l'ordre 
établi, suppose in fine de décrire les rapports de pouvoir, leur régulation et les ressources afférentes. La démarche de proximité a été utilisée pour décrire comment les acteurs se partageaient un espace géographique et des ressources cognitives - représentations, valeurs, normes, règles, etc. - pour agir collectivement. Elle doit l'être aussi pour souligner que les acteurs se répartissent inégalement les ressources du pouvoir, ressources qui sont autant de moyens de peser sur les comportements des partenaires de l'interaction (Braud, 2008). L'analyse de la répartition inégale d'actifs financiers, du contrôle de l'information, de la notoriété, de la légitimité, etc. comme pré-requis à l'action collective reste à faire dans l'approche de la proximité. Alors la dimension politique y sera totalement intégrée.

\section{Bibliographie}

Amable B., Palombarini S., 2004. Une approche néo-réaliste de l'économie politique. Douzième rencontre internationales du GERPISA, Comment penser la variété du capitalisme et la diversité des modèles productifs, juin, Paris.

Amable B., Palombarini S., 2005. L'économie politique n'est pas une science morale. Raisons d'agir Editions, Paris.

Bazzoli L., 1999. L'économie politique de John R. Commons. L'Harmattan, Paris.

Bazzoli L., Kirat T., 1996. La relation entre le droit et l'économie dans les traditions institutionnaliste et post-coasienne: enjeux pour l'analyse évolutionniste. Colloque Évolutionnisme, fondements, perspectives et réalisations, Paris Sorbonne, METIS, septembre.

Bellet M., Colletis G., Lung Y, (eds), 1993. Economie de proximités. Revue d'Economie Régionale et Urbaine, $\mathrm{n}^{\circ} 3$.

Boschma R., 2005. Does geographical proximity favour innovation? Economie et Institutions, 6 et 7, 111-126.

Bouba-Olga O., Grossetti M., 2008. Socio-économie de proximité. Revue d'Economie Régionale et Urbaine, 3, 311-328.

Bouba-Olga O., Carrincazeaux C., Coris M., (eds), 2008. La proximité : 15 ans déjà ! Revue d'Economie Régionale et Urbaine, $\mathrm{n}^{\circ} 3$.

Bourdeau-Lepage L., Huriot J.M., 2009. Proximités et interactions : une reformulation. Géographie, Economie, Société, 11, 3, 233-249.

Braud P., 2008. Sociologie politique. $9^{\mathrm{e}}$ édition, Lextenso éditions, Paris.

Carrincazeaux C., Lung Y., 1998. La proximité dans l'organisation des activités de conception des produits automobiles. M. Bellet, T. Kirat et C. Largeron (eds), Approches multiformes de la proximité, Hermès, Paris.

Carrincazeaux C., Grossetti M., Talbot D., (eds) 2008. Clusters, proximities and networks. European Planning Studies, vol.16, ${ }^{\circ} 5$.

Carrincazeaux C., Lung Y., Vicente J., 2008. The scientific trajectory of the french school of proximity: interaction- and institution- based approaches to Regional System of Innovation. European Planning Studies, 16, 5, 617-628. 
Colletis G., Pecqueur B., 2005. Révélation de ressources spécifiques et coordination située. Economie et Institutions, 6 et 7, 51-74.

Commons J.R., 1931. Institutional Economics. American Economic Review, December, 648657.

Commons J.R., 1934. Institutional Economics. Its Place in Political Economy. MacMillan Company, New York.

Dubet F., 2009. Le travail des sociétés. Editions du Seuil, Paris.

Eymard-Duvernay F., 2001. Pour un programme d'économie institutionnaliste. Colloque AFSE, septembre.

Fligstein N., 1996. Markets as politics: a political-cultural approach to market institutions. American Sociological Review, 61, 4, 656-673.

Gilly J.P., Torre A., (eds), 2000. Dynamiques de proximité. L'Harmattan, Paris.

Grossetti M., Bes M.P., 2001. Interacting individuals and organizations: a case study on cooperations between firms and research laboratories. A. Kirman et J.B. Zimmermann (eds), Economics with heterogeneous interacting agents, Springer, Berlin.

Hassenteufel P., 1995. Les groupes d'intérêt dans l'action publique : l'Etat en interaction. Pouvoirs, 74, 155-168.

Hassenteufel P., 2010. Les processus de mise sur agenda : sélection et construction des problèmes publics. Informations sociales, 157, 50-58.

Jullien B., Smith A., (eds), 2008. Industries and Globalization. The Political Causality of Divergent Responses. Palgrave, London.

Kirat T., Lung Y., 1995. Innovations et proximités : le territoire, lieu de déploiement des processus d'apprentissage. N. Lazaric et J. M. Monnier (eds.), Coordination économique et apprentissage des firmes, Economica, Paris.

Kirat T., Torre A., (eds), 2008. Territoires de conflits. Analyses des mutations de l'occupation de l'espace. L'Harmattan, Paris.

Laganier R., Villalba B., Zuindeau Z., 2002. Le développement durable face au territoire : éléments pour une recherche pluridisciplinaire. Revue Développement Durable et Territoires, dossier $\mathrm{n}^{\circ} 1$ Approches territoriales du Développement Durable, téléchargeable sur http://developpementdurable.revues.org/sommaire105.html

Lagroye J., 2003. Les processus de politisation. J. Lagroye (ed.), La politisation, Belin, Paris.

Lagroye J., François B., Sawicki F., 2006. Sociologie Politique, 5 édition, Presses de Sciences Po et Dalloz, Paris.

Le Breton D., 2004. L'interactionnisme symbolique. Presses Universitaires de France, Paris.

Lussault M., 2007. L'homme spatial. La construction sociale de l'espace humain. Collection « la couleur des idées », Seuil, Paris.

Mauss M., 1950. Sociologie et Anthropologie. Presses Universitaires de France, $7^{\mathrm{e}}$ édition premier trimestre 1980, Paris.

Muller P., 2005. Esquisse d'une théorie du changement dans l'action publique. Revue Française de Science Politique, 55, 1, 155-184.

Muller P., 2008. Les politiques publiques. $7^{\mathrm{e}}$ édition, Presses Universitaires de France, Paris.

Pecqueur B., Zimmermann J.B., 2004. Introduction. Les fondements d'une économie de proximités. B. Pecqueur B., et J.B. Zimmermann, (eds), Economie de Proximités, Hermès, Lavoisier, Paris.

Perroux F., 1973. Pouvoir et Économie. Dunod, Paris.

Rallet A., Torre A., 2007. Introduction. Faut-il être proche pour innover ensemble? A. Rallet et A. Torre (eds), Quelles proximités pour innover?, L'Harmattan, Paris.

Rychen F., Zimmermann J.B., (eds), 2008. Clusters in the Global Knowledge-based Economy: Knowledge Gatekeepers and Temporary Proximity. Special Issue of Regional Studies, 42, 6, 767-776. 
Scott R., 2001. Institutions and Organizations. Second Edition, Sage Publications, London, $255 \mathrm{p}$.

Searle J., 1998. La construction de la réalité sociale. Gallimard, Paris.

Searle J., 2005. What is an institution? Journal of Institutional Economics, 1, 1, 1-22.

Suire R., Vicente J., 2008. Théorie économique des clusters et management des réseaux d'entreprises innovantes. Revue française de gestion, 4, 184, 119-136.

Talbot D., Kirat T., (eds), 2005. Proximité et Institutions : nouveaux éclairages. Economie et Institutions, $\mathrm{n}^{\circ} 6$ et 7,1 er et $2 \mathrm{e}$ semestres.

Talbot D., 2008a. Les institutions créatrices de proximités. Revue d'Economie Régionale et Urbaine, 3, 289-310.

Talbot D., 2008b. EADS, une transition inachevée. Une lecture par les catégories de la proximité. Géographie, Economie, Société, 10, 2, 243-261.

Torre A., 2009. Retour sur la notion de proximité géographique. Géographie, Economie, Société, 11, 63-75.

Torre A., Caron A., 2005. Réflexions sur les dimensions négatives de la proximité : le cas des conflits d'usages et de voisinages. Economie et Institutions, ${ }^{\circ} 6$ et $7,1 \mathrm{er}$ et $2 \mathrm{e}$ semestres, 183-220.

Torre A., Rallet A., 2005. Proximity and localization, Regional Studies. 39, 1, 47-60.

Torre A., Zuindeau B., 2009. Les apports de l'économie de la proximité aux approches environnementales : inventaire et perspectives. Natures Sciences Sociétés, 17, 349-360. 